PARALLEL MEASUREMENT AND MODELING OF TRANSPORT IN THE DARHT II BEAMLINE ON ETA II

F. W. Chambers, B. A. Raymond, S. Falabella, B. S. Lee, R. A. Richardson, J. T. Weir, H. A. Davis, M. E. Schultze

June 1, 2005

IEEE International Pulsed Power Conference Monterey, CA, United States June 13, 2005 through June 17, 2005 
This document was prepared as an account of work sponsored by an agency of the United States Government. Neither the United States Government nor the University of California nor any of their employees, makes any warranty, express or implied, or assumes any legal liability or responsibility for the accuracy, completeness, or usefulness of any information, apparatus, product, or process disclosed, or represents that its use would not infringe privately owned rights. Reference herein to any specific commercial product, process, or service by trade name, trademark, manufacturer, or otherwise, does not necessarily constitute or imply its endorsement, recommendation, or favoring by the United States Government or the University of California. The views and opinions of authors expressed herein do not necessarily state or reflect those of the United States Government or the University of California, and shall not be used for advertising or product endorsement purposes. 


\title{
PARALLEL MEASUREMENT AND MODELING OF TRANSPORT IN THE DARHT II BEAMLINE ON ETA II*
}

\author{
Frank W. Chambers, Brett A. Raymond, Steven Falabella, Bryan S. Lee, Roger A. \\ Richardson, and John T. Weir \\ Lawrence Livermore National Laboratory \\ Livermore, California \\ Harold A. Davis ${ }^{\ddagger}$ \\ Martin E. Schultze ${ }^{\dagger}$
}

\begin{abstract}
To successfully tune the DARHT II transport beamline requires the close coupling of a model of the beam transport and the measurement of the beam observables as the beam conditions and magnet settings are varied. For the ETA II experiment using the DARHT II beamline components this was achieved using the SUICIDE (Simple User Interface Connecting to an Integrated Data Environment) data analysis environment and the FITS (Fully Integrated Transport Simulation) model. The SUICIDE environment has direct access to the experimental beam transport data at acquisition and the FITS predictions of the transport for immediate comparison. The FITS model is coupled into the control system where it can read magnet current settings for real time modeling. We find this integrated coupling is essential for model verification and the successful development of a tuning aid for the efficient convergence on a useable tune. We show the real time comparisons of simulation and experiment and explore the successes and limitations of this close coupled approach.
\end{abstract}

\section{INTRODUCTION}

Particle accelerators use magnetic transport to propagate the electron beam from the accelerator to the target. In the case of the DARHT II accelerator a discrete set of solenoids and quadrupoles performs this transport. In the design phase of the project magnet locations, profiles, and field strengths are determined based on various design transport codes ${ }^{1}$. However, when the accelerator becomes operational the adjustment or tuning of this transport line will be required.

For high rep rate, low energy accelerators where many reproducible pulses are available and there are sufficient diagnostics the tuning process can be accomplished by the operator using his/her understanding of transport and the feedback received from each machine shot. The Experimental Test Accelerator II (ETA II) at Lawrence Livermore National Laboratory with a pulse rate of $1 \mathrm{~Hz}$, energy of $5 \mathrm{MeV}$, current of $\sim 2 \mathrm{kA}$, and pulse duration of 50ns meets the majority of these criteria although diagnostics are relatively sparse. The DARHT II accelerator with a pulse rate in the neighborhood of 3 pulses/h, energy of $\sim 18 \mathrm{MeV}$, current $\sim 2 \mathrm{kA}$, and pulse duration of $2 \mu$ sec cannot be tuned in this manner.

A more practical approach for transport tuning in this environment is to employ a transport model closely coupled to the transport as a tool for operator guidance. In the recent experiments with the DARHT II transport beamline deployed on the ETA II accelerator ${ }^{2}$ this approach was attempted using the LAMDA code ${ }^{3}$ and the FITS code for the modeling. This paper reports the successes and failures of the FITS modeling experience.

\section{FITS Model of the DARHT beamline in SUICIDE}

The portion of the DARHT beamline to be modeled starts at an aperture plate which defines the beam radius and limits the current transmitted. After this there are two solenoids, five quadrupoles, and then two further solenoids leading to the target as illustrated in Figure 1. Insertible foils on the sketch show where optical data on beam size is available.

The FITS (Fully Integrated Transport Simulation) model is a beam transport model with a graphical user interface and copious diagnostics which runs under the SUICIDE (Simple User Interface Connecting to an Integrated Data Environment ${ }^{4}$ ) environment. The beam dynamics model is selectable from several options including envelope models and particle models with various dynamics effects included or excluded. For the transport of the beam through quadrupoles the particle models are used exclusively.

\footnotetext{
* This work was performed under the auspices of the U.S. Department of Energy by University of California, Lawrence Livermore National Laboratory under Contract W-7405-Eng-48.
} 


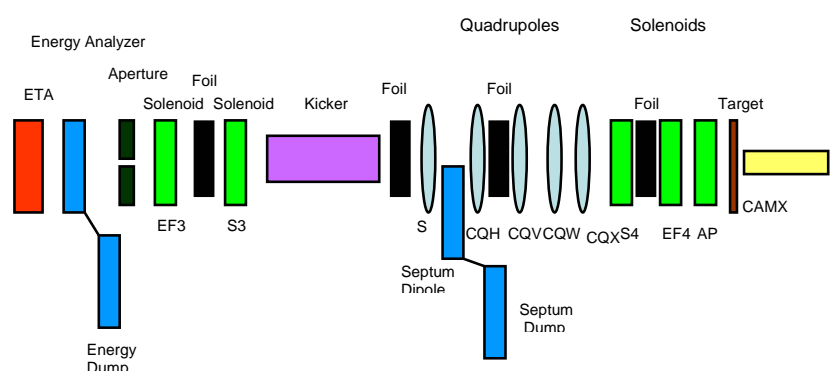

Figure 1. Sketch of the DARHT II transport beamline as deployed at ETA II.

The FITS model provides for the calculation of beam transport for a single configuration and for the calculation of transport with parameter values swept through a prescribed space. It is capable of searching through a multiple parameter space to match observed beam radii with FITS predictions. This capability is used to determine beam injection conditions.

The FITS model is closely coupled to the ETA II accelerator in that it reads the magnet current directly from the control system. The user can grab the magnet tune and then vary one magnet to get a prediction of the consequences of this variation. (FITS can not actually control magnets (YET) because of operator reluctance and safety concerns).

FITS has been used extensively to determine the beam parameters at injection. An early FITS triumph was the demonstration that the beam energy was $\sim 5.0 \mathrm{MeV}$ and not the $5.8 \mathrm{MeV}$ previously believed. Since this was a model prediction it required a year of digestion and two other energy measurements to convince experimentalists that indeed FITS was correct.

We have found that for transport through a single magnetic element FITS agreement with experiment can be made excellent. However, when multiple elements are involved the accumulation of uncertainties renders the predictions problematic.

\section{Beam Size Data}

The primary connection between theory and experiment is the beam size. There are many code diagnostics used to parameterize beam radius and diameter. For envelope and particle modeling the RMS radius is preferred while other transport codes utilize an edge radius.

The observed beam size is usually determined from analysis of light generated by the beam striking an insertible probe in the beamline. These images are characterized by several uncertainties, the most significant being the question of optical background at large radii. Diagnostics which are sensitive to this background level include the RMS radius (the favorite of envelope modelers) and the MTF or Modulation Transfer Function radius (favored by radiographers). Varying assumed background levels can change the calculate radius by up to $\sim 50 \%$.

For these experiments the beam RMS radius is calculated from the digitally captured beam image by fitting a rotated two dimensional Gaussian to the smoothed, cropped image. This gives a major radius, minor radius, and angle of rotation. The fit determines a background or base level for the image.

Although this is a well defined algorithm which produces reasonable results it must be acknowledged that other reasonable interpretations are possible. The beam radius measurement for large beams is further complicated by the finite size of the insertible probe and the presence of return current bars which shadow the image. For small beams the light generation mechanism on the probe is in question leading to further ambiguity. In spite of these concerns the RMS radius as determined above will be used for comparison with the models.

\section{BEAM ENTRANCE CONDITIONS}

The modeling of beam transport requires that the beam entrance conditions to the transport section and the mechanical/magnetic configuration of the transport section be well characterized.

Beam entrance conditions include the macroscopic parameters such as the current, I; the radius, R; the rate of change of the radius, R'; the emittance, $\varepsilon$; and the energy, E. The actual microscopic distribution of particles in phase space may be important but is not measured simple Gaussian or flat distributions are assumed.

For the DARHT on ETA beamline the entrance point is defined at an aperture where the beam current is reduced from $\sim 1800 \mathrm{~A}$ to $\sim 600$ amps. This aperture has an opening of 0.75 " diameter which for a flat profile translates to $R_{R M S}=0.6735 \mathrm{~cm}$. The beam current $\mathrm{I}$ is determined by the next beambug after the aperture. The beam energy $E$ is based on energy analyzer measurement rescaled to account for the FITS observations of the actual lower energy than implied by the historical calibration of the analyzer.

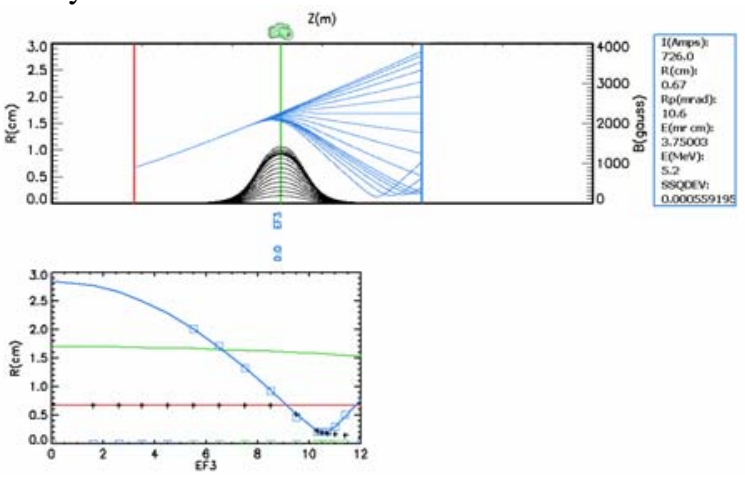

Figure 1. Determination of beam entrance condition by FITS modeling of an EF3 magnet scan.

The remaining beam entrance conditions of R' and $\varepsilon$ are determined by scanning the magnetic field of the next solenoid and observing the beam size on the next available popin. The FITS run showing the agreement between prediction and data is shown in Figure 1. The large R' was of concern because the beam was tuned to a 
minimum size at the aperture and an R' of 0 was anticipated. A FITS scan of entrance conditions addressing the aperture demonstrated that the minimum beam size on aperture was not a waist but rather a significantly diverging beam, see Figure 3 .

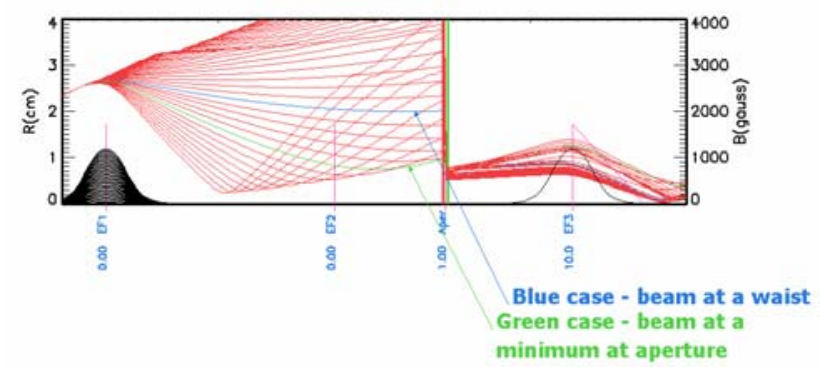

Figure 2. Entrance conditions showing how beam is at a minimum but not at a waist.

\section{Transport and Predictions - Few Magnets}

As the beam was being brought online a number of prediction of magnet values were made to assist the operator in setting individual magnets. Transport through the first two solenoids was well predicted. A tune was developed in FITS to transport the beam through the quadrupoles. Indeed the transport of a round beam was observed at about this setting. We were able to predict tunes reasonably well which produced a round beam at the end of this transport. When solenoid magnet currents were varied we could predict the rate of rotation induced in an oblong beam from the quadrupoles. The closely coupled FITS model aided in the initial transport of the beam down the pipe.

A particularly impressive success came when we were completely unable to predict the beam size versus S4 magnet current. The focusing occurred at a current approximately $50 \%$ above that predicted by FITS. After considerable speculation we examined the magnet and found that many of the multiple power/water connections on the magnet were shorted together thereby reducing the field to $2 / 3$ of the predicted value. The magnet was fixed at that time and then agreed with FITS predictions.

\section{Transport and Predictions - Multiple Magnets}

Attempts to model in detail the full transport through the entire magnet system (including solenoids and quadrupoles only; excluding steering, kicking, and septupoles) met with far more limited success. Due to the sparseness of diagnostics often single component effects could not be isolated. With only one magnetic element energized the beam at the next diagnostic port would be too large to measure properly. With multiple elements energized the performance in general was correctly modeled but the actual details of the modeling were not so successful.

The problem is illustrated with one scan of a quadrupole magnet in figures 4 and 5 . When quadrupoles are varied the beam varies from oblong to round to oblong at 90 degrees rotation from the original ellipse as seen in figure 4. This behavior was indeed observed but the currents at which the specific profiles (e.g. circular) were observed did not agree with the predictions. Moreover the beam sizes at these significant points were quite different from predictions as seen in Figure 5.

This inadequacy of the model to predict multicomponent transport requires an investigation of the sources of error.

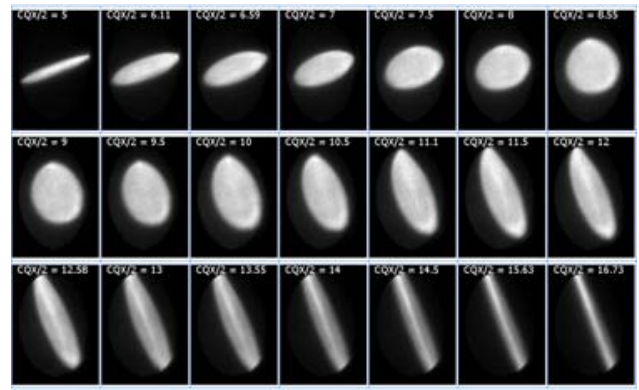

Figure 3. Scan of the CQX quadrupole showing the characteristic behavior of ellipse - circular - ellipse.

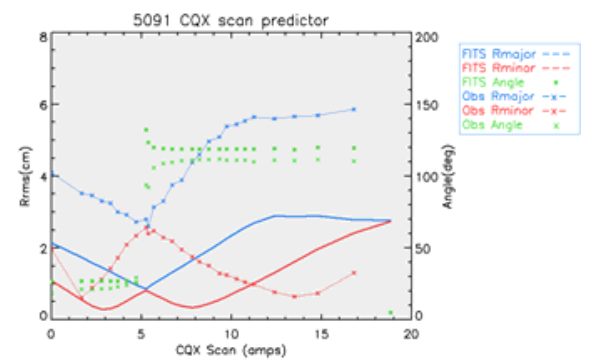

Figure 4. Comparison of CQX scan and FITS prediction showing agreement in nature but markedly different major and minor radii at each current.

\section{Limitation of Transport Predictions} A. Magnets

A primary source of error is the magnetic field of the the transport magnets. The ETA II control system allows the operator to set a Request value for the magnet current and then it provides a Readback value of the supposed magnet current. Knowing the Request/Readback values allows the operator to return to a particular recorded tune. Unfortunately; these values do not accurately represent the actual currents in the magnets. In specific instances either the Readback or the Requested values were close to the actual values. In other cases neither value was correct. Late in the experiment a linear least square fits were performed to remove this ambiguity. Unfortunately the errors in current measurements were found to be not repeatable. The notion of correcting bad diagnostics by calibrations after the experiments is fundamentally flawed, these problems must be addressed prior to operations. This problem potentially introduces error in the $\sim 5 \%$ level.

The quadrupole magnets contained iron and therefore had residual fields which were not measured and therefore 
not compensated for. Prescriptions were put forth for minimizing these field but with insufficient current available on the power supplies this could not be implemented. The errors introduced are estimated as $\sim 5 \%$.

The magnet profiles were based on model calculations performed prior to construction of the magnets. Subsequently the model and measured profiles have been compared. While they are quite close errors in the $\sim 1 \%$ range can be present - particularly in the case of ETA II where the magnets were being operated at lower than anticipated currents due to the lower beam energy.

Finally magnet installation was not always in accord with the initial design. The S4 was installed without the endcap due to constraints on the pipe size. This field distortion is calculated to be $<\sim 1 \%$.

In short there are multiple small problems with the magnetic field calculation (even without higher order terms) which compromise the ability to predict magnetic transport.

\section{B. Beam}

The beam entrance conditions are determined from observations of beam propagation with the first solenoid magnet scanned. These conditions are then used for the experiment on subsequent days. The presumption of constant entrance conditions is entirely not justified for the ETA II operating conditions, variations of upwards of $\sim 5 \%$ may be present.

Only the macroscopic beam parameters are measured with the FITS fitting technique. However, microscopic details such as the true beam profile, energy distribution, velocity distribution may influence the transport. Various models within FITS have been run and for most cases remarkable agreement between models is observed but there is always some slight divergence; say $\sim 2 \%$. This is especially true for an expanding beam such as is being launched for these experiments.

Oftentimes one can obtain agreement between the model and observation by arbitrarily adjusting entrance conditions. While this is at times gratifying it constitutes a postdiction and not a prediction of beam behavior.

\section{Model}

The beam dynamics models in the FITS code have very real limitations. The envelope model is only good for $\mathrm{m}=0$ propagation and cannot transport a beam through quadrupoles. The particle models have a primitive implementation of space charge. All models are "single disk" and ignore the head to tail beam and field evolution.

To validate or vilify the models one must pursue comparisons with other models as well as data. A detailed comparison with the LAMDA code is begin pursued preliminary comparisons showed agreement in principle but detailed comparisons are required.
As noted earlier the diagnostics for determining beam size have significant limitations in particular for large or small beams or images with significant background levels (that is - all images). A key parameter for comparison of code and measurement for quadrupole scans is the current at which the beam is round - the determination of roundness depends on the calibration of the orientation of the insertible probe which varies with each insertion. Beam orientation errors range in the $5 \%$ arena.

\section{Conclusions}

Multiple problems with these experiments have been identified and solutions can be proposed. However there is a fundamental question of the "predictability" of beam transport which must be asked. A large number of small uncertainties in the dynamics of beam transport have been identified. One can systematically try to reduce these uncertainties. However; there may be fundamental limitations on the predictability of the complex system. This problem is reminiscent of weather prediction where the forecast for one day (one magnet) is pretty good but the seven day (seven magnet) prediction is not.

One tack is to try to reduce the uncertainties highlighted in section VII for the four areas mentioned. After completion of this series of experiments we have begun to explore this course with an improved optical diagnostic probe. If we can model the transport we can tune with a limited number of diagnostic ports.

Another view is that the model will give qualitative guidance to the tuner but not a quantitative predictive capability. The approach as previously outlined ${ }^{1}$ starts with a "predicted" tune but then provides the operator primarily with guidance as to which knob to turn and in what direction rather then giving a definitive setpoint. This approach will require more shots on target than a fully calibrated model would necessitate.

Present efforts are focused on determining where the predictive shortfalls occur and to what extent they can be remedied. We will continue to research ways to improve the predictive capability. If the opportunity arises we will perform additional experiments; particularly if better magnet control / readout can be achieved. Simultaneously we are reviewing the heuristic algorithms to be employed for optimal convergence on appropriate DARHT II transport tunes.

References:

[1] Art Paul

[2] John Weir

[3] Tom Hughes

[4] Frank Chambers

${ }^{\ddagger}$ Los Alamos National Laboratory, Los Alamos, New
$\quad$ Mexico
${ }^{\dagger}$ General Atomics, Los Alamos, New Mexico

\section{Diagnostics}

